J. Clin. Chem. Clin. Biochem.

Vol. 15, 1977, pp. 213-216

\title{
Simultane Bestimmung von freiem Cholesterin und Gesamtcholesterin auf dem Technicon AA I Autoanalyser
}

\author{
Von C. C. Heuck und A. Wirth
}

Klinisches Institut für Herzinfarktforschung, Heidelberg

(Eingegangen am 18. Mai/26. August 1976)

Zusammenfassung: Es wird die enzymatische Bestimmung von freiem Cholesterin und Gesamtcholesterin im Serum auf einem 2-Kanal Autoanalyser AA I Technicon System beschrieben. Die Bestimmung des freien Cholesterins wird mit der gaschromatographischen Bestimmung von freiem Cholesterin verglichen. Wie die Untersuchung zeigt, werden nach beiden Verfahren aus menschlichem Serum nahezu gleiche Meßwerte gefunden. Gegenüber den herkömmlichen Bestimmungsmethoden von freiem Cholesterin hat das Verfahren den Vorteil, daß es schnell und einfach arbeitet.

\section{Simultaneous determination of free cholesterol and total cholesterol on an AA I Technicon autoanalyzer}

Summary: A procedure for the simultaneous enzymatic determination of free cholesterol and total cholesterol on a 2 channel AA I Technicon autoanalyzer system is described. The quantitation of free cholesterol is compared with the determination by gas chromatography. The investigation reveals a high correlation between both methods. In contrast to classical procedures the proposed quantitation of free cholesterol can be performed easily and quickly for routine analyses.

\section{Einleitung}

Das Verhältnis der Konzentrationen von freiem Cholesterin zu Gesamtcholesterin im Serum beträgt normalerweise $1 / 4$ bis $1 / 3$. Es kann jedoch bei Funktionsstörungen der Leber, bei genetischen Stoffwechselerkrankungen oder Störungen des Hormonhaushaltes sich zugunsten des freien Cholesterins verschieben. Bisher wurde von der Bestimmung von freiem Cholesterin als einem Parameter in der Verlaufskontrolle eines Krankheitsbildes in der Klinik wenig Gebrauch gemacht, weil die herkömmlichen Bestimmüngsmethoden für die Routineanalytik zu aufwendig sind, wie ż. B. die Gaschromatographie (1) oder die Dünnoschichtchromatógraphie (2) und Fällungsreaktionen mit Digitonin (3) sich als ungenau erwiesen. Im Folgenden soll ein Verfahren zur enzymatischen Bestimmung von freiem Cholesterin alis dem Serum mit dem Autoanalyser vorgestellt werden. Es arbeitet einfach und schnell und - wie der Vergleich mit der gaschromatographischen Bestimmung von freiem Cholesterin zeigt - auch mit ausreichender Präzision.

\section{Material und Methodik}

Die enzymatische Bestimmung von freiem Cholesterin und Gesamtcholesterin wurde auf einem AA I Technicon Autoanalyser System nach dem Fließschema, das in Abbildung 1 wiedergegeben ist, durchgefürt. Parallel zu dem Fließsystem für die Bestimmung von Gesamtcholesterin wurde ein System zur Bestimmung des freien Cholesterins aufgebaut. Die photometrische Absorptionsmessung erfolgte bei $505 \mathrm{~nm}$.

Parallel zu der enzymatischen Bestimmung nach der CHODPAP $^{1}$ ) Methode wurde das Gesamtcholesterin zum Vergleich auf einem AA II Technicon Autoanalyser chemisch aus dem Isopropanolextrakt nạch Liebermann-Burchard gemessen (5). Die Kalibrierung des Autoanalysers erfolgte mit einem Serum, dessen Cholesteringehalt nach der Abell-Kendall-Methode bestimmt worden war (6).

Die gaschromatographischen Bestimmungen wurden auf einem Hewlett-Packard A 5830-Gerät mit Autosampler und HewlettPackard Terminal auf einer SE 30-Säule (Gaschrom Q 125 mesh, Säulentemperatur $240^{\circ} \mathrm{C}$, Stickstoffluß $51 \mathrm{~mol} / \mathrm{min}$ ) ausgefuhrt (4).

\footnotetext{
1) Unüblichẹ Abkürzung: CHOD-PAP = CholesterinoxidasePhenol-4-Aminophenazon.
} 


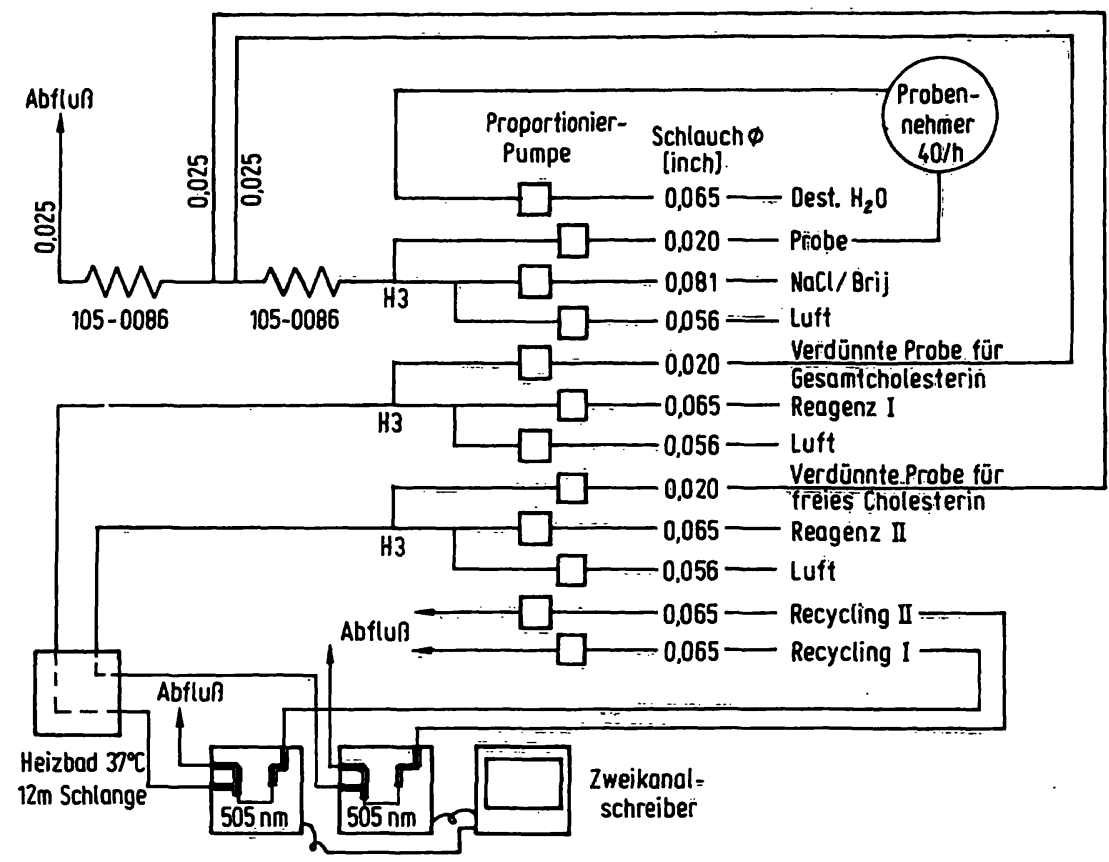

Fig. 1. Fließdiagramm zur enzymatischen Simultanbestimmung von Gesamtcholesterin und freiem Cholesterin auf dem AA I TECHNICON Autoanalyzer. Reagenz I ist die Lösung zur Bestimmung von Gesamtcholesterin nach der CHOD-PAP-

Methode (BOEHRINGER, Mannheim), Reagenz II enthält die gleichen Reagenzien wie Reagenz I außer Cholesterinesterase. Die Konzentration an Meerrettichperoxidase beträgt 2,5 U/1. Die Verdünnungslösung $(0,325 \mathrm{~mol} / 1 \mathrm{NaCl} / 0,3 \%$ Brij 35$)$ entspricht der Firmenangabe zur Bestimmung von Cholesterin nach der CHOD-PAP-Methode (BOEHRINGER, Mannheim).

a

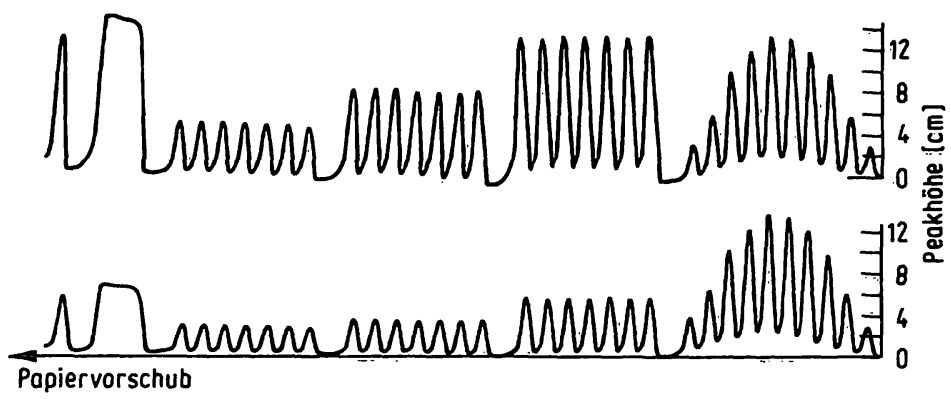

b

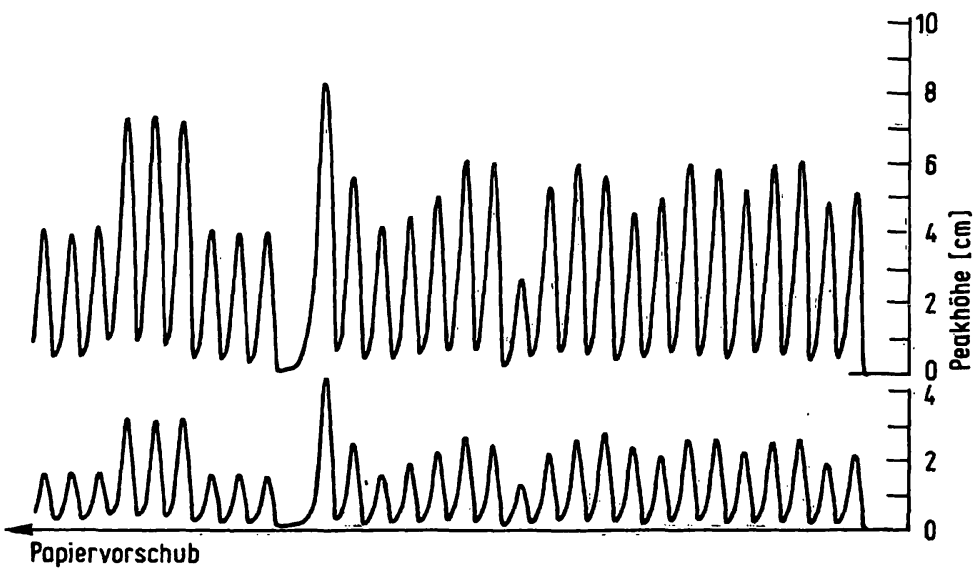

Fig. 2. a) Simultane Registration der Standardkurve (Standardkonzentrationen: $1,29 \mathrm{mmol} / 1,2,59 \mathrm{mmol} / 1,5,18 \mathrm{mmol} / 1,7,77 \mathrm{mmol} / \mathrm{l}$, $10,36 \mathrm{mmol} / \mathrm{l}$ freies Cholesterin in Isopropanol) sowie von Gesamtcholesterin und freiem Cholesterin aus 3 verschiedenen Seren und einem Dauerlauf.

b) Registration der Konzentrationen von Gesamtcholesterin und freiem Cholesterin aus 20 verschiedenèn Seren, sowie des carry over. 


\section{Reagenzien}

Testkombination zur Bestimmung von Gesamtcholesterin nach der CHOD-PAP-Methode (7), Packung (Boehringer Mannheim, Germany). Zur Bestimmung des freien Cholesterins wurde die Cholesterinoxidase, Phenol und das Farbreagenz 4-Aminophenazon aus dem gleichen Bestimmungsset übernommen. Zusätzlich wurde Meerrettichperoxidase, gereinigt (Packung 15302, Boehringer Mannheim, Germany), die keine enzymatische Aktivität einer Cholinesterase aufwies, eingesetzt. Die Konzentration der Peroxidaselösung betrug 2,5 U/1. DieStandardlösungen wurden mit freiem Cholesterin (gaschromatographisch rein, SERVA Heidelberg, Germany) in Isopropanol p. a. (Merck, Darmstadt) (Standardreihe: $1,3 \mathrm{mmol} / 1,259 \mathrm{mmol} / \mathrm{l}, 5,8 \mathrm{mmol} / 1$, $7,77 \mathrm{mmol} / 1,10,36 \mathrm{mmol} / \mathrm{l}$ ) angesetzt.

Die Seren wurden von Patienten erhalten, die sich stationär in der Univ.-Klinik für Innere Medizin, Heidelberg, aufhielten. Bei den enzymatischen Messungen.wurden die Seren unvorbehandelt weiter verarbeitet. Für die gaschromatographischen Untersuchungen wurden 0,5 ml Serum mit $9,5 \mathrm{ml}$ Isopropanol p. a. in Gegenwart von Zeolit extrahiert (5). $1 \mathrm{ml}$ des Isopropanol-Extraktes wurden $20 \mu$ l einer $\alpha$-Cholestan-Lösung $(2,68 \mathrm{mmol} / 1$ Ethanol) als internem Standard für die Gaschromatographie zugesetzt. Die gaschromatographische Quantifizierung erfolgte aus $1 \mu \mathrm{l}$ dieser Lösung.

Die Präzision der enzymatischen Bestimmung von freiem Cholesterin wurde aus 20 Messungen eines Serums ermittelt. Die Präzision von Tag zu Tag wurde aus einem Serum, das in aliquoten Portionen tiefgefroren aufbewahrt wurde, gemessen. Über einen Zeitraum von 4 Wochen wurde täglich eine Probe zur Verlaufskontrolle aufgetaut.

\section{Ergebnisse}

Die simultane Bestimmung von Gesamtcholesterin und freiem Cholesterin ist in Abbildung 2 wiedergegeben. Als Standardlösung wurde freies Cholesterin in Isopropanol gemessen. Wie der Verlauf der Kurvèn żeigt, werden erwartungsgemäß für beide Messungen gleich hohe Absorptionen erreicht. Im Gegensatz hierzu werden auf dem Kanal für freies Cholesterin aus 3 Seren mit einem niederen $(2,20 \mathrm{mmol} / \mathrm{l})$, einem mittleren $(6,53$ mmol/1) und einem hohen $(10,49 \mathrm{mmol} / \mathrm{l})$ Gesamtcholesterinwert nur etwa $1 / 3$ dieser Werte erreicht. Bei einem Verhältnis der Waschphase/Probenphase von 2:1 kann sowohl bei der Bestimmung für Gesamtcholesterin wie für freies Cholesterin ein carry over von hohen Cholesterinkỏnzentrationen zu niederen Cholesterinkonzentrationeñ auf weniger als $2 \%$ dés zweiten Wertes beschränkt werden (Abb. 2a).

Auf dem Technicon AA I-System verläuft die Standardkurve bis zú 2,59 mmol/1 linear, um dann bei höheren Werten abzuflachen (Abb. 3).

Die Präzision wurde aus 20 aliquoten Messungen eines Serums bestimmt. Bei einem Mittelwert von 6,97 $\pm 0,10$ $\mathrm{mmol} / \mathrm{l}(\overline{\mathrm{x}} \pm \mathrm{s})$ Gesamtcholesterin ergibt sich ein Variationskoeffizient von $\mathrm{VK}=1,4$, für einen Mittelwert von $1,66 \pm 0,03 \mathrm{mmol} / 1(\mathrm{x} \pm \mathrm{s})$ freiem Cholesterin ist der entsprechende Variationskoeffizient $\mathrm{VK}=2,0$. In einer Verlaufskontrolle wurde fự das gleiche Serum über einen Z̈eitraum von 20 Tagen ein Variationskoeffizient $\mathrm{VK}=4,4$ ermittelt.

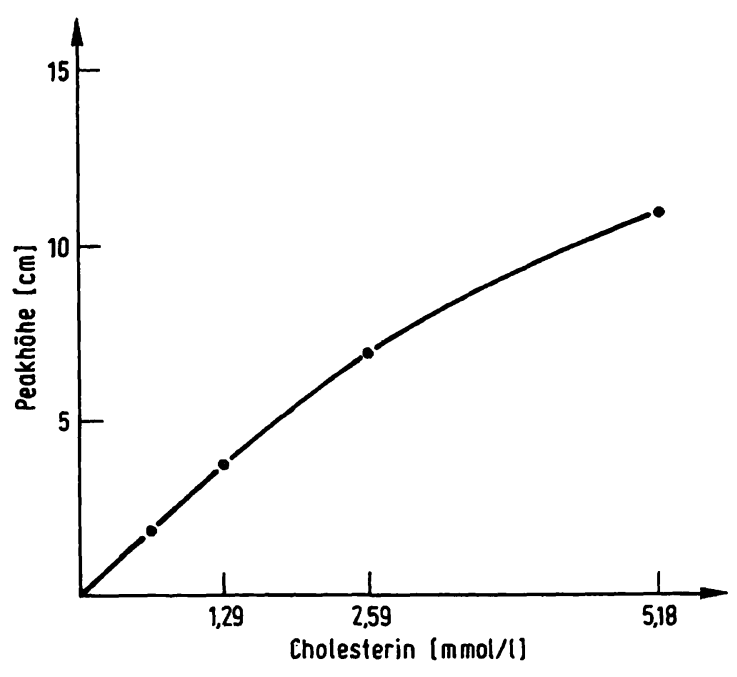

Fig. 3. Standardkurve von Cholesterin in Isopropanol (Transmissionsmessung mit Hilfe eines Technicon-Kolorimeters der 1 . Generation).

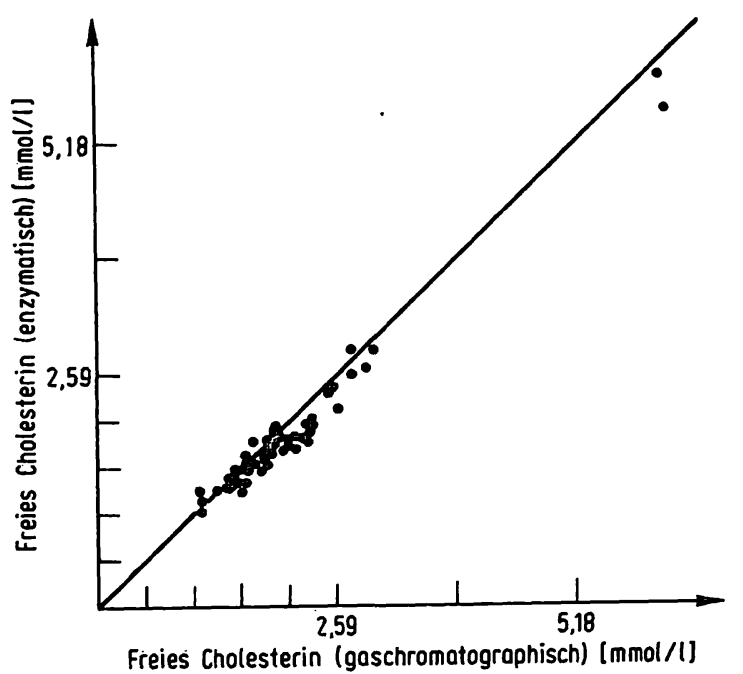

Fig. 4. Korrelation zwischen der enzymatischen Bestimmung von freiem Cholesterin im Serum $(n=67)$ auf dem Autoanalyzer und der gaschromatographischen Bestimmung. Regressionsgerade:

$y_{\text {enzymatisch }}=0,947 \cdot x_{\text {gaschromatographisch }}-0,889$; $r=0,988$.

Der Vergleich der enzymatischen Bestimmung und der gaschromatographischen Bestimmung von freiem Cholesterin wurde an 67 Seren durchgeführt. Bei einer Korrelation von $\mathrm{r}=0,988$ liegen die Meßwerte für die enzymatische Bestimmung etwa um 3-5\% unter den gaschromatographisch gemessenen Werten in einem Bereich von $1,04 \mathrm{mmol} / 1$ bis $6,22 \mathrm{mmol} / 1$ freiem Cholesterin (Abb. 4). Die Relation Gesamtcholesterin/ freies Cholesterin wurde einerseits aus den Absorptionen der enzymatischen Bestimmungen (Abb. 2a), andererseits aus der gaschromatographischen Messung von freiem Cholesterin und der chemischen Bestimmung von Gesamtcholesterin nach Liebermann-Burchard errechnet. In der Tabelle 1 sind die Relationen aus Seren von Normolipämikern, Hyperlipoproteinämikern und 
Tab. 1. Vergleich der enzymatischen und chemischen Bestimmung von freiem Cholesterin und Gesamtcholesterin in Seren von Normolipämikern, Hyperlipoproteinämikern (Typ II) und Hepatitis-Patienten.

\begin{tabular}{|c|c|c|c|c|c|c|}
\hline & $\begin{array}{l}\text { enzymati } \\
\text { Gesamt- } \\
\text { chole- } \\
\text { sterin } \\
\text { [mmol/1] }\end{array}$ & $\begin{array}{l}\text { freies } \\
\text { Chole- } \\
\text { sterin } \\
\text { [mmol/1] }\end{array}$ & $\begin{array}{l}\text { Ver- } \\
\text { hält- } \\
\text { nis }\end{array}$ & $\begin{array}{l}\text { chemisch- } \\
\text { graphisch } \\
\text { Gesamt- } \\
\text { chole- } \\
\text { sterin } \\
\text { [mmol/l] }\end{array}$ & $\begin{array}{l}\text {-gaschroma } \\
\text { freies } \\
\text { Chole- } \\
\text { sterin } \\
\text { [mmol/l] }\end{array}$ & $\begin{array}{l}\text { lato- } \\
\text { Ver- } \\
\text { hält- } \\
\text { nis }\end{array}$ \\
\hline $\begin{array}{l}\text { Normoli- } \\
\text { pämiker }\end{array}$ & $\begin{array}{l}3,76 \\
549 \\
4,53 \\
6,21 \\
2,85 \\
6,13 \\
6,09 \\
6,22 \\
6,40 \\
6,94\end{array}$ & $\begin{array}{l}1,09 \\
1,48 \\
1,35 \\
1,99 \\
1,06 \\
1,68 \\
1,74 \\
1,97 \\
1,82 \\
1,99\end{array}$ & $\begin{array}{l}3,38 \\
3,71 \\
3,36 \\
3,11 \\
2,68 \\
3,64 \\
3,50 \\
3,15 \\
3,43 \\
3,48\end{array}$ & $\begin{array}{l}3,81 \\
5,52 \\
4,77 \\
5,49 \\
3,32 \\
6,29 \\
6,35 \\
6,60 \\
6,42 \\
7,07\end{array}$ & $\begin{array}{l}1,11 \\
1,53 \\
1,48 \\
1,92 \\
1,11 \\
1,81 \\
1,79 \\
2,28 \\
1,89 \\
1,92\end{array}$ & $\begin{array}{l}3,41 \\
3,61 \\
3,22 \\
2,86 \\
2,97 \\
3,47 \\
3,55 \\
2,89 \\
3,39 \\
3,68\end{array}$ \\
\hline $\begin{array}{l}\text { Typ II } \\
\text { a/b }\end{array}$ & $\begin{array}{l}8,81 \\
9,58 \\
8,29 \\
8,68 \\
9,32 \\
7,54 \\
9,97 \\
8,94 \\
8,94 \\
7,25 \\
7,59\end{array}$ & $\begin{array}{l}1,94 \\
2,59 \\
2,07 \\
2,25 \\
2,43 \\
2,43 \\
2,46 \\
2,54 \\
2,12 \\
2,23 \\
2,12\end{array}$ & $\begin{array}{l}4,50 \\
3,70 \\
4,00 \\
3,85 \\
3,82 \\
3,09 \\
4,05 \\
3,61 \\
4,20 \\
3,25 \\
3,57\end{array}$ & $\begin{array}{l}8,26 \\
9,45 \\
7,61 \\
8,65 \\
9,07 \\
8,16 \\
9,32 \\
8,44 \\
8,88 \\
7,33 \\
8,11\end{array}$ & $\begin{array}{l}1,89 \\
2,75 \\
2,12 \\
2,43 \\
2,51 \\
2,51 \\
2,56 \\
2,59 \\
2,28 \\
2,31 \\
2,46\end{array}$ & $\begin{array}{l}4,36 \\
3,44 \\
3,58 \\
3,55 \\
3,60 \\
3,24 \\
3,63 \\
3,26 \\
3,89 \\
3,17 \\
3,29\end{array}$ \\
\hline $\begin{array}{l}\text { Hepa- } \\
\text { titis }\end{array}$ & $\begin{array}{l}2,62 \\
3,57 \\
2,98 \\
3,83 \\
4,61 \\
5,05 \\
4,17 \\
7,12 \\
2,69\end{array}$ & $\begin{array}{l}1,55 \\
2,20 \\
2,51 \\
2,28 \\
3,63 \\
1,94 \\
1,81 \\
4,92 \\
1,42\end{array}$ & $\begin{array}{l}1,68 \\
1,62 \\
1,18 \\
1,68 \\
1,27 \\
2,60 \\
2,30 \\
1,44 \\
1,89\end{array}$ & $\begin{array}{l}2,62 \\
4,07 \\
3,11 \\
3,29 \\
4,71 \\
5,10 \\
4,09 \\
7,38 \\
2,64\end{array}$ & $\begin{array}{l}1,66 \\
2,36 \\
2,38 \\
2,43 \\
3,70 \\
2,10 \\
1,86 \\
4,69 \\
1,58\end{array}$ & $\begin{array}{l}1,60 \\
1,76 \\
1,30 \\
1,35 \\
1,24 \\
2,43 \\
2,19 \\
1,57 \\
1,67\end{array}$ \\
\hline
\end{tabular}

Patienten mit Leberfunktionsstörungen wiedergegeben. Nach beiden Bestimmungsverfahren wurden nahezu gleiche Ergebnisse erhalten.

\section{Diskussion}

In der Untersuchung sollte festgestellt werden, ob freies Cholesterin aus dem Serum enzymatisch bestimmt werden kann. Der Vergleich zwischen der gaschromatographischen Messung und der enzymatischen Bèstimmung zeigt, daß nach beiden Verfahren im Rahmen der Fehlerbreite der Bestimmung die gleichen Werte erhalten werden. Daraus kann gefolgert werden, daß die enzymatische Bestimmung des freien Cholesterins aus dem Serum unter der Voraussetzung möglich ist, dạß cholesterinesterasefreie Enzympräparationen verwendet werden. Andernfalls würden durch zusätzliche Hydrolyse von Cholesterinester überhöhte Werte gemessen werden.

Die Bestimmung des Cholesterins in den Standardlösungen auf dem Autoanalyzer zeigt, daß die oxidative Kupplung von P̈henol mit 4-Aminophenażon bei der Bestimmung von freiem Cholesterin die gleichen Absorptionswerte ergibt wie für die Mèssung von Gesamtcholesterin. Die Farbstoffbildung läuft demnach bei der Bestimmung des freien Cholesterins ebenso unbeeinträchtigt ab wie bei der Bestimmung des Geșamtcholesterins.

Die simultane Messung von Gesamtcholesterin und freiem Cholesterin auf einem 2-Kanal Autoanalyzer System vereinfacht die Bestimmung des relativen Anteils an freiem Cholesterin im Serum. Sie bietet eine Möglichkeit zu routinemäßigen Verlaufskontrollen, die wegen der herkömmlichen aufwendigen Verfahren in der klinischen Praxis nur beschränkt durchgeführt worden sind.

\section{Literatur}

1. Schmitt, J. A., Wynne, R. B. \& Mather, A. (1964), F \& M Scientific Corp. Bull. No. 116.

2. Ganshirt, H. (1967), Quantitative Auswertung von Dünnschichtchromatogrammen; Dünnschichtchromatographie (E. Stahl, ed.) 2. Auflage S. 137, Springer Verlag, Berlin, Heidelberg, New York.

3. Huang, T. C., Wefler, W. \& Raftery, A. (1963), Anal. Chem. 35, 1757-1758.

4. Heuck, C. C., Nothhelfer, A., Raetzer, H. \& Schlierf, G., J. Lipid Res. for publication.

5. Technicon clinical method No. 24 (1972), Technicon Instruments, Tarrytown, N. Y.

6. Abell, L. L., Blevy, B., Brodie, B. \& Kendall, R. E. (1952), J. Biol. Chem. 195, 357.

7. Klose, S., Greif, H. \& Hagen, A. (1975), 9. Intern. Congress on clinical chemistry Abstract 8.
Dr. Dr. C. Heuck Bergheimer Str. 58 D-6900 Heidelberg 